\title{
New Exact Solutions of Ion-Acoustic Wave Equations by $\left(G^{\prime} / G\right)$-Expansion Method
}

\author{
Wafaa M. Taha, M. S. M. Noorani, and I. Hashim \\ School of Mathematical Sciences, Universiti Kebangsaan Malaysia UKM, 43600 Bangi, Selangor, Malaysia \\ Correspondence should be addressed to Wafaa M. Taha; wafaa_y2005@yahoo.com
}

Received 24 May 2013; Revised 19 September 2013; Accepted 20 September 2013

Academic Editor: Saeid Abbasbandy

Copyright (C) 2013 Wafaa M. Taha et al. This is an open access article distributed under the Creative Commons Attribution License, which permits unrestricted use, distribution, and reproduction in any medium, provided the original work is properly cited.

The $\left(G^{\prime} / G\right)$-expansion method is used to study ion-acoustic waves equations in plasma physic for the first time. Many new exact traveling wave solutions of the Schamel equation, Schamel-KdV (S-KdV), and the two-dimensional modified KP (KadomtsevPetviashvili) equation with square root nonlinearity are constructed. The traveling wave solutions obtained via this method are expressed by hyperbolic functions, the trigonometric functions, and the rational functions. In addition to solitary waves solutions, a variety of special solutions like kink shaped, antikink shaped, and bell type solitary solutions are obtained when the choice of parameters is taken at special values. Two- and three-dimensional plots are drawn to illustrate the nature of solutions. Moreover, the solution obtained via this method is in good agreement with previously obtained solutions of other researchers.

\section{Introduction}

The ion-acoustic solitary wave is one of the fundamental nonlinear waves phenomena appearing in fluid dynamics [1] and plasma physics [2]. To allowing for the trapping of some of the electrons on ion-acoustic waves, Schamel proposed a modified equation for ion-acoustic waves [3] given by

$$
u_{t}+u^{1 / 2} u_{x}+\delta u_{x x x}=0,
$$

where $u$ is the wave potential and $\delta$ is a constant, this equation describing the ion-acoustic wave, where the electrons do not behave isothermally during their passage of the wave in a cold-ion plasma. Then, combining the equations of Schamel and the $\mathrm{KdV}$ equation, one obtains the so-called onedimensional form of the Schamel-KdV (S-KdV) equation equation $[4,5]$ :

$$
u_{t}+\left(\alpha u^{1 / 2}+\beta u\right) u_{x}+\delta u_{x x x}=0, \quad \delta \beta \neq 0,
$$

where $\beta, \alpha$, and $\delta$ are constants. This equation is established in plasma physics in the study of ion acoustic solitons when electron trapping is present, and also it governs the electrostatic potential for a certain electron distribution in velocity space. Note that we obtain the KdV equation when $\alpha=0$ and the Schamel equation when $\beta=0$ for $\delta=1$. Due to the wide range of applications of (2), it is important to find new exact wave solutions of the Schamel-KdV (S$\mathrm{KdV}$ ) equation. Another equation arising in the study of ion-acoustic waves is the so-called modified KadomtsevPetviashvili (KP) equation given by [6]

$$
\left(u_{t}+\alpha u^{1 / 2} u_{x}+\beta u_{x x x}\right)_{x}+\delta u_{y y}=0 .
$$

Equation (3) was firstly derived by Chakraborty and Das [7]; the modified KP equation containing a square root nonlinearity is a very attractive model for the study of ionacoustic waves in a multispecies plasma made up of nonisothermal electrons in plasma physics.

In the literature, the KP equation is also known as the twodimensional KdV equation [8].

It has lately become more interesting to obtain exact analytical solutions to nonlinear partial differential equations such as the one arising from the ion-acoustic wave phenomena, by using appropriate techniques. Several important techniques have been developed such as the tanh-method [9, 10], sine-cosine method [11, 12], tanh-coth method [13], expfunction method [14], homogeneous-balance method [15, 16], 
Jacobi-elliptic function method [17, 18], and first-integral method $[19,20]$ to solve analytically nonlinear equations such as the above ion-acoustic wave equations.

Moreover, in the standard tanh method developed by Malfliet in 1992 [21], the tanh is used as a new variable. Since all derivatives of a tanh are represented by tanh itself, the solution obtained by this method may be solitons in terms of $\operatorname{sech}^{2}$ or may be kinks in terms of tanh. We believe that the $\left(G^{\prime} / G\right)$-expansion method is more efficient than the tanh method. Moreover, the tanh method may yield more than one soliton solution, a capability which the tanh method does not have. The sine-cosine method yields a solution in trigonometric form. The Exp-function method leads to both generalized solitary solution and periodic solutions. The homogeneous-balance method is a generalized tanh function method for many nonlinear PDEs. The first integral method, which is based on the ring theory of commutative algebra, was first proposed by Feng. There is no general theory telling us how to find its first integrals in a systematic way; so, a key idea of this approach to find the first integral is to utilize the division theorem. The traveling wave solutions expressed by the $\left(G^{\prime} / G\right)$-expansion method, which was first proposed by Wang et al. [22], transform the given difficult problem into a set of simple problems which can be solved easily to get solutions in the forms of hyperbolic, trigonometric, and rational functions. The main merits of the $\left(G^{\prime} / G\right)$-expansion method over the other methods are as follows.

(i) Higher-order nonlinear equations can be reduced to ODEs of order greater than 3.

(ii) There is no need to apply the initial and boundary conditions at the outset. The method yields a general solution with free parameters which can be identified by the above conditions.

(iii) The general solution obtained by the $\left(G^{\prime} / G\right)$-expansion method is without approximation.

(iv) The solution procedure can be easily implemented in Mathematica or Maple.

In fact, the $\left(G^{\prime} / G\right)$-expansion method has been successfully applied to obtain exact solution for a variety of NLPDE [23-34].

In this paper, the $\left(G^{\prime} / G\right)$-expansion method is used to study ion-acoustic waves equations in plasma physic for the first time. We obtain many new exact traveling wave solutions for the Schamel equation, S-KdV, and the two-dimensional modified KP equation. The traveling wave solutions obtained via this method are expressed by hyperbolic functions, the trigonometric functions, and the rational functions. In addition to solitary waves solutions, a variety of special solutions like kink shaped, antikink shaped, and bell type solitary solutions are obtained when the choice of parameters is taken at special values. Two- and three-dimensional plots are drawn to illustrate the nature of solutions. Moreover, the solution obtained via this method is in good agreement with previously obtained solutions of other researchers.
Our paper is organized as follows: in Section 2, we present the summary of the $\left(G^{\prime} / G\right)$-expansion method, and Section 3 describes the applications of the $\left(G^{\prime} / G\right)$-expansion method for Schamel equation, S-KdV equation, and modified KP equation, and lastly, conclusions are given in Section 4.

\section{Summary of the $\left(G^{\prime} / G\right)$-Expansion Method}

In this section, we describe the $\left(G^{\prime} / G\right)$-expansion method for finding traveling wave solutions of NLPDE. Suppose that a nonlinear partial differential equation in two independent variables, $x$ and $t$, is given by

$$
p\left(u, u_{t}, u_{x}, u_{x t}, u_{t t}, u_{x x}, \ldots\right)=0
$$

where $u=u(x, t)$ is an unknown function, $P$ is a polynomial in $u=u(x, t)$ and its various partial derivatives, in which highest order derivatives and nonlinear terms are involved.

The summary of the $\left(G^{\prime} / G\right)$-expansion method can be presented in the following six steps.

Step 1. To find the traveling wave solutions of (4), we introduce the wave variable:

$$
u(x, t)=u(\zeta), \quad \zeta=(x-c t)
$$

where the constant $c$ is generally termed the wave velocity. Substituting (5) into (4), we obtain the following ordinary differential equations (ODE) in $\zeta$ (which illustrates a principal advantage of a traveling wave solution; i.e., a PDE is reduced to an ODE):

$$
p\left(u, c u^{\prime}, u^{\prime}, c u^{\prime \prime}, c^{2} u^{\prime \prime}, u^{\prime \prime}, \ldots\right)=0
$$

Step 2. If necessary, we integrate (6) as many times as possible and set the constants of integration to be zero for simplicity.

Step 3. Suppose that the solution of nonlinear partial differential equation can be expressed by a polynomial in $\left(G^{\prime} / G\right)$ as

$$
u(\zeta)=\sum_{i=0}^{m} a_{i}\left(\frac{G^{\prime}}{G}\right)^{i}
$$

where $G=G(\zeta)$ satisfies the second-order linear ordinary differential equation

$$
G^{\prime \prime}(\zeta)+\lambda G^{\prime}(\zeta)+\mu G(\zeta)=0
$$

where $G^{\prime}=d G / d \zeta, G^{\prime \prime}=d^{2} G / d \zeta^{2}$, and $a_{i}, \lambda$, and $\mu$ are real constants with $a_{m} \neq 0$. Here, the prime denotes the derivative with respect to $\zeta$. Using the general solutions of (8), 
we have

$$
\begin{aligned}
& \left(\frac{G^{\prime}}{G}\right)
\end{aligned}
$$

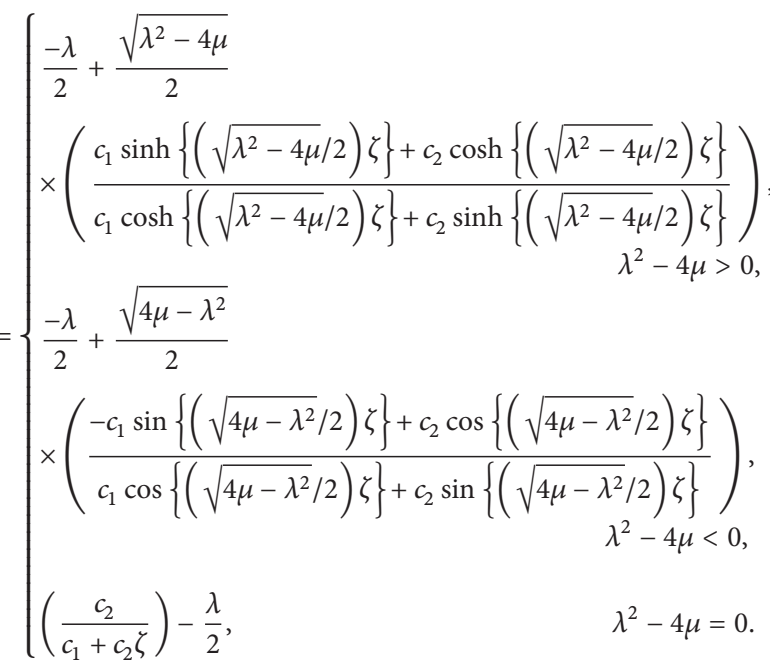

The above results can be written in simplified forms as

$$
\begin{aligned}
& \left(\frac{G^{\prime}}{G}\right) \\
& = \begin{cases}\frac{-\lambda}{2}+\frac{\sqrt{\lambda^{2}-4 \mu}}{2} \tanh \left\{\frac{\sqrt{\lambda^{2}-4 \mu}}{2} \zeta\right\}, & \lambda^{2}-4 \mu>0, \\
\frac{-\lambda}{2}+\frac{\sqrt{4 \mu-\lambda^{2}}}{2} \tan \left\{\frac{\sqrt{4 \mu-\lambda^{2}}}{2} \zeta\right\}, & \lambda^{2}-4 \mu<0, \\
\left(\frac{c_{2}}{c_{1}+c_{2} \zeta}\right)-\frac{\lambda}{2}, & \lambda^{2}-4 \mu=0 .\end{cases}
\end{aligned}
$$

Step 4. The positive integer $m$ can be accomplished by considering the homogeneous balance between the highest order derivatives and nonlinear terms appearing in (6) as follows: if we define the degree of $u(\zeta)$ as $D[u(\zeta)]=m$, then the degree of other expressions is defined by

$$
\begin{gathered}
D\left[\frac{d^{q} u}{d \zeta^{q}}\right]=m+q, \\
D\left[u^{r}\left(\frac{d^{q} u}{d \zeta^{q}}\right)^{s}\right]=m r+s(q+m) .
\end{gathered}
$$

Therefore, we can get the value of $m$ in (7).

Step 5. Substituting (7) into (6), useing general solutions of $(8)$, and collecting all terms with the same order of $\left(G^{\prime} / G\right)$ together, then setting each coefficient of this polynomial to zero yields a set of algebraic equations for $a_{i}, c, \lambda$, and $\mu$.

Step 6. Substitute $a_{i}, c, \lambda$, and $\mu$ obtained in Step 5 and the general solutions of (8) into (7). Next, depending on the sign of the discriminant $A=\lambda^{2}-4 \mu$, we can obtain the explicit solutions of (4) immediately.

\section{Applications of the $\left(G^{\prime} / G\right)$-Expansion Method}

3.1. Schamel Equation. In order to find the solitary wave solution of (1), we use the transformations

$$
u(x, t)=v^{2}(x, t), \quad v(x, t)=v(\zeta), \quad \zeta=k x-c t
$$

Then, (1) becomes

$$
-c v v^{\prime}+k v^{2} v^{\prime}+\delta k^{3}\left(v v^{\prime \prime \prime}+3 v^{\prime} v^{\prime \prime}\right)=0 .
$$

Integrating (13) with respect to $\zeta$ and setting the integration constant equal to zero, we have

$$
-\frac{c}{2} v^{2}+\frac{k}{3} v^{3}+k^{3} \delta\left(v^{\prime}\right)^{2}+k^{3} \delta v v^{\prime \prime}=0
$$

According to the previous steps, using the balancing procedure between $v^{3}$ with $v v^{\prime \prime}$ in (14), we get $3 m=2 m+2$ so that $m=2$. Now, assume that (14) has the following solution:

$$
v(\zeta)=a_{0}+a_{1}\left(\frac{G^{\prime}}{G}\right)+a_{2}\left(\frac{G^{\prime}}{G}\right)^{2}, \quad a_{2} \neq 0,
$$

where $a_{0}, a_{1}$, and $a_{2}$ are unknown constants to be determined later. Substituting (15) along with (8) into (14) and collecting all terms with the same order of $\left(G^{\prime} / G\right)$, the left hand side of (14) is converted into a polynomial in $\left(G^{\prime} / G\right)$. Equating each coefficient of the resulting polynomials to zero yields a set of algebraic equations for $a_{0}, a_{1}, a_{2}, \delta, \lambda, c, k$, and $\mu$ as follows:

$$
\begin{gathered}
\left(\frac{G^{\prime}}{G}\right)^{6}: \frac{1}{3} k a_{2}^{3}+10 k^{3} \delta a_{2}^{2}=0, \\
\left(\frac{G^{\prime}}{G}\right)^{5}: 18 k^{3} \delta a_{2}^{2} \lambda+12 k^{3} \delta a_{1} a_{2}+k a_{1} a_{2}^{2}=0, \\
\left(\frac{G^{\prime}}{G}\right)^{4}: 6 k^{3} \delta a_{0} a_{2}+16 k^{3} \delta a_{2}^{2} \mu+8 k^{3} \delta a_{2}^{2} \lambda^{2}-\frac{1}{2} c a_{2}^{2} \\
+21 k^{3} \delta a_{1}^{2}+k a_{0} a_{2}^{2}+k a_{1}^{2} a_{2}=0, \\
\left(\frac{G^{\prime}}{G}\right)^{3}: 14 k^{3} \delta a_{2}^{2} \lambda \mu+5 k^{3} \delta a_{1}^{2} \lambda+18 k^{3} \delta a_{1} a_{2} \mu \\
+9 k^{3} \delta a_{1} \lambda^{2} a_{2}+2 k a_{0} a_{1} a_{2}-c a_{1} a_{2}+\frac{1}{3} k a_{1}^{3} \\
+2 k^{3} \delta a_{0} a_{1}+10 k^{3} \delta a_{0} a_{2} \lambda=0,
\end{gathered}
$$




$$
\begin{gathered}
\left(\frac{G^{\prime}}{G}\right)^{2}: 2 k^{3} \delta a_{1}^{2} \lambda^{2}+4 k^{3} \delta a_{1}^{2} \mu+8 k^{3} \delta a_{0} a_{2} \mu-\frac{1}{2} c a_{1}^{2}-c a_{0} a_{2} \\
+15 k^{3} \delta a_{1} a_{2} \lambda \mu+k a_{0} a_{1}^{2}+4 k^{3} \delta a_{0} a_{2} \lambda^{2} \\
+6 k^{3} \delta a_{2}^{2} \mu^{2}+3 k \delta a_{0} a_{1} \lambda+k a_{0}^{2}=0 \\
\left(\frac{G^{\prime}}{G}\right)^{1}: 2 k^{3} a_{0} a_{1} \mu+k^{3} \delta a_{0} a_{1} \lambda^{2}+6 k^{3} \delta a_{0} a_{2} \lambda \mu+k a_{0}^{2} \\
-c a_{0} a_{1}+6 k^{3} a_{1} a_{2} \delta \mu^{2}+3 k^{3} \delta \lambda \mu a_{1}^{2}=0 \\
\left(\frac{G^{\prime}}{G}\right)^{0}: k^{3} \delta a_{1}^{2} \mu^{2}-\frac{1}{2} c a_{0}^{2}+\frac{1}{3} k a_{0}^{3}+k^{3} \delta \lambda \mu a_{0} a_{1} \\
+2 k^{3} \delta \mu^{2} a_{0} a_{2}=0 .
\end{gathered}
$$

On solving the above set of algebraic equations by Maple, we have

$$
\begin{gathered}
a_{0}=-30 \mu \delta k^{2}, \quad a_{1}=-30 k^{2} \delta \lambda, \\
a_{2}=-30 k^{2} \delta, \quad c=\delta k^{3}\left(4 \lambda^{2}-16 \mu\right) .
\end{gathered}
$$

Now, (15) becomes

$$
v(\zeta)=-30 \mu \delta k^{2}-30 k^{2} \delta \lambda\left(\frac{G^{\prime}}{G}\right)-30 k^{2} \delta\left(\frac{G^{\prime}}{G}\right)^{2} .
$$

Substituting the general solution of (8) into (18), we obtain the three types of traveling wave solutions depending on the sign of $A=\lambda^{2}-4 \mu$.

If $A>0$, we have the following general hyperbolic traveling wave solutions of (1):

$$
\begin{aligned}
& v(x, t) \\
& =-30 \mu \delta k^{2}-30 k^{2} \delta \lambda \\
& \times\left[\frac{-\lambda}{2}+\frac{\sqrt{A}}{2}\right. \\
& \left.\times\left(\frac{c_{1} \sinh \{(\sqrt{A} / 2) \zeta\}+c_{2} \cosh \{(\sqrt{A} / 2) \zeta\}}{c_{1} \cosh \{(\sqrt{A} / 2) \zeta\}+c_{2} \sinh \{(\sqrt{A} / 2) \zeta\}}\right)\right] \\
& -30 k^{2} \delta\left[\frac{-\lambda}{2}+\frac{\sqrt{A}}{2}\right. \\
& \left.\quad \times\left(\frac{c_{1} \sinh \{(\sqrt{A} / 2) \zeta\}+c_{2} \cosh \{(\sqrt{A} / 2) \zeta\}}{c_{1} \cosh \{(\sqrt{A} / 2) \zeta\}+c_{2} \sinh \{(\sqrt{A} / 2) \zeta\}}\right)\right]^{2},
\end{aligned}
$$

where $c_{1}$ and $c_{2}$ are arbitrary constants.
If $A<0$, we have the following general trigonometric function solutions of (1):

$$
\begin{aligned}
& v(x, t) \\
& =-30 \mu \delta k^{2}-30 k^{2} \delta \lambda \\
& \times\left[\frac{-\lambda}{2}+\frac{\sqrt{A}}{2}\right. \\
& \left.\times\left(\frac{-c_{1} \sin \{(\sqrt{A} / 2) \zeta\}+c_{2} \cos \{\sqrt{A} / 2\}}{c_{1} \cos \{(\sqrt{A} / 2) \zeta\}+c_{2} \sin \{(\sqrt{A} / 2) \zeta\}}\right)\right] \\
& -30 k^{2} \delta\left[\frac{-\lambda}{2}+\frac{\sqrt{A}}{2}\right. \\
& \left.\times\left(\frac{-c_{1} \sin \{(\sqrt{A} / 2) \zeta\}+c_{2} \cos \{(\sqrt{A} / 2) \zeta\}}{c_{1} \cos \{(\sqrt{A} / 2) \zeta\}+c_{2} \sin \{(\sqrt{A} / 2) \zeta\}}\right)\right]^{2} .
\end{aligned}
$$

If $A=0$, we have the following general rational function solutions of (1):

$$
\begin{aligned}
v(x, t)= & -30 \mu \delta k^{2}-30 k^{2} \delta \lambda\left[-\frac{\lambda}{2}+\left(\frac{c_{2}}{c_{1}+c_{2} \zeta}\right)\right] \\
& -30 k^{2} \delta\left[-\frac{\lambda}{2}+\left(\frac{c_{2}}{c_{1}+c_{2} \zeta}\right)\right]^{2},
\end{aligned}
$$

where $\zeta=k x-\delta k^{3}\left(4 \lambda^{2}-16 \mu\right) t$.

Writing $u(x, t)=v^{2}(x, t)$ and setting $c_{2}=\mu=0$ and $\lambda=2$ in (19), we reproduce the result of Khater and Hassan [35] (see their Equation (4.7)),

$$
u(x, t)=4\left(900 k^{4} \delta^{2} \operatorname{sech}^{4}\{\zeta\}\right),
$$

where $\zeta=k x-16 \delta k^{3} t$.

Note that Khater and Hassan [35] obtained only hyperbolic solutions, but in this work, we found two additional types of solutions, that is, trigonometric and rational solutions.

3.2. S-KdV Equation. To find the general exact solutions of (2), we first write $u(x, t)=v^{2}(x, t)$ to transform (2) into

$$
v v_{t}+\left(\alpha v+\beta v^{2}\right) v_{x}+\delta v v_{x x x}=0 .
$$

Assume the traveling wave solution of (23) in the form

$$
v(x, t)=V(\zeta), \quad \zeta=k(x-c t) .
$$

Hence, (23) becomes

$$
-c V V^{\prime}+\left(\alpha V^{2}+\beta V^{3}\right) V^{\prime}+k^{2} \delta\left(V V^{\prime \prime \prime}+3 V^{\prime} V^{\prime \prime}\right)=0 .
$$

Suppose that the solution of (25) can be expressed by a polynomial in $\left(G^{\prime} / G\right)$ as

$$
V(\zeta)=\sum_{i=0}^{m} a_{i}\left(\frac{G^{\prime}}{G}\right)^{i}, \quad a_{i} \neq 0
$$


and $G(\zeta)$ satisfies (8). The homogeneous balance between the highest order derivative $V V^{\prime \prime \prime}$ and the nonlinear term $V^{3} V^{\prime}$ appearing in (25) yields $m=1$, and hence, we take the following formal solution:

$$
V(\zeta)=a_{0}+a_{1}\left(\frac{G^{\prime}}{G}\right)
$$

where the positive integers $a_{0}$ and $a_{1}$ are to be determined later. Substituting (27) along with (8) into (25), collecting all the terms with the same power of $\left(G^{\prime} / G\right)$, and equating each coefficient to zero yield a set of simultaneous algebraic equations for $a_{0}, a_{1}, c, k, \alpha, \beta$, and $\delta$ as follows:

$$
\begin{aligned}
& \left(\frac{G^{\prime}}{G}\right)^{5}:-\beta a_{1}^{4}-12 \delta k^{2} a_{1}^{2}=0, \\
& \left(\frac{G^{\prime}}{G}\right)^{4}:-3 \beta a_{1}^{3} a_{0}-\beta a_{1}^{4} \lambda-27 \delta k^{2} a_{1}^{2} \lambda-\alpha a_{1}^{3} \\
& -6 \delta k^{2} a_{0} a_{1}=0 \\
& \left(\frac{G^{\prime}}{G}\right)^{3}:-3 \beta a_{1}^{3} \lambda a_{0}-19 \delta k^{2} a_{1}^{2} \lambda^{2}-12 \delta k^{2} a_{0} a_{1} \lambda \\
& -20 \delta k^{2} a_{1}^{2} \mu-\alpha a_{1}^{3} \lambda-\beta a_{1}^{4} \mu+c a_{1}^{2}-2 \alpha a_{1}^{2} a_{0} \\
& -3 \beta a_{1}^{2} a_{0}^{2}=0, \\
& \left(\frac{G^{\prime}}{G}\right)^{2}: c a_{0} a_{1}-3 \beta a_{1}^{2} \lambda a_{0}^{2}-2 \alpha a_{1}^{2} \lambda a_{0}+c a_{1}^{2} \lambda-26 \delta k^{2} a_{1}^{2} \lambda \mu \\
& -8 \delta k^{2} a_{0} a_{1} \mu-7 \delta k^{2} a_{0} a_{1} \lambda^{2}-3 \beta a_{1}^{3} \mu a_{0}-\beta a_{1} a_{0}^{3} \\
& -\alpha a_{1} a_{0}^{2}-\alpha a_{1}^{3} \mu=0, \\
& \left(\frac{G^{\prime}}{G}\right)^{1}:-\alpha \lambda a_{1} a_{0}^{2}+c a_{0} a_{1} \lambda-8 \delta k^{2} a_{1}^{2} \mu^{2}-7 \delta \mu k^{2} a_{1}^{2} \lambda^{2} \\
& -3 \beta \mu a_{1}^{2} a_{0}^{2}+c a_{1}^{2} \mu-\beta \lambda a_{1} a_{0}^{3}-8 \delta \lambda \mu k^{2} a_{0} a_{1} \\
& -2 \alpha \mu a_{1}^{2} a_{0}=0 \text {, } \\
& \left(\frac{G^{\prime}}{G}\right)^{0}: c \mu a_{0} a_{1}-\alpha \mu a_{1} a_{0}^{2}-\beta \mu a_{1} a_{0}^{3}-3 \delta \lambda k^{2} a_{1}^{2} \mu^{2} \\
& -\delta \mu \lambda^{2} k^{2} a_{0} a_{1}-2 \delta \mu^{2} k^{2} a_{0} a_{1}=0 .
\end{aligned}
$$

The above system admits the following sets of solutions:

$$
\begin{gathered}
a_{0}=0, \quad a_{1}=\frac{4 \alpha}{5 \beta \lambda}, \quad \mu=0, \\
c=-\frac{16 \alpha^{2}}{75 \beta}, \quad k= \pm \frac{2 \sqrt{1 /-75 \delta \beta} \alpha}{\lambda},
\end{gathered}
$$

$$
\begin{gathered}
a_{0}=-\frac{4 \alpha}{5 \beta}, \quad a_{1}=-\frac{4 \alpha}{5 \beta \lambda}, \quad \mu=0, \\
c=-\frac{16 \alpha^{2}}{75 \beta}, \quad k= \pm \frac{2 \sqrt{1 /-75 \delta \beta} \alpha}{\lambda}, \\
a_{0}=\frac{5 \beta a_{1} \lambda-4 \alpha}{10 \beta}, \quad \mu=\frac{25 \beta^{2} a_{1}^{2} \lambda^{2}-16 \alpha^{2}}{100 \beta^{2} a_{1}^{2}}, \\
c=-\frac{16 \alpha^{2}}{75 \beta}, \quad k= \pm \sqrt{\frac{\beta}{-12 \delta} a_{1} .}
\end{gathered}
$$

Now, substituting (29)-(30) into (27) gives, respectively,

$$
\begin{gathered}
V_{1}(\zeta)=\frac{4 \alpha}{5 \beta \lambda}\left(\frac{G^{\prime}}{G}\right), \\
V_{2}(\zeta)=-\frac{4 \alpha}{5 \beta}-\frac{4 \alpha}{5 \beta \lambda}\left(\frac{G^{\prime}}{G}\right), \\
V_{3}(\zeta)=\frac{5 \beta a_{1} \lambda-4 \alpha}{10 \beta}+a_{1}\left(\frac{G^{\prime}}{G}\right) .
\end{gathered}
$$

When substituting the general solutions (9) into (32), we obtain the following three types of traveling wave solutions.

Case $A>0$ : (hyperbolic type)

$$
\begin{gathered}
v_{1}(x, t)=-\frac{2 \alpha}{5 \beta}+\frac{2 \alpha}{5 \beta}\left(\frac{c_{1} \sinh \{(\lambda / 2) \zeta\}+c_{2} \cosh \{(\lambda / 2) \zeta\}}{c_{1} \cosh \{(\lambda / 2) \zeta\}+c_{2} \sinh \{(\lambda / 2) \zeta\}}\right), \\
\zeta= \pm \frac{2 \sqrt{1 /-75 \delta \beta} \alpha}{\lambda} x+\frac{16 \alpha^{2}}{75 \beta} t, \\
v_{2}(x, t)=-\frac{2 \alpha}{5 \beta}-\frac{2 \alpha}{5 \beta}\left(\frac{c_{1} \sinh \{(\lambda / 2) \zeta\}+c_{2} \cosh \{(\lambda / 2) \zeta\}}{c_{1} \cosh \{(\lambda / 2) \zeta\}+c_{2} \sinh \{(\lambda / 2) \zeta\}}\right), \\
\zeta= \pm \frac{2 \sqrt{1 /-75 \delta \beta} \alpha}{\lambda} x+\frac{16 \alpha^{2}}{75 \beta} t,
\end{gathered}
$$$$
v_{3}(x, t)
$$$$
=-\frac{2 \alpha}{5 \beta}+\frac{2 \alpha}{5 \beta}
$$

$$
\times\left(\frac{c_{1} \sinh \left\{\left(2 \alpha / 5 \beta a_{1}\right) \zeta\right\}+c_{2} \cosh \left\{\left(2 \alpha / 5 \beta a_{1}\right) \zeta\right\}}{c_{1} \cosh \left\{\left(2 \alpha / 5 \beta a_{1}\right) \zeta\right\}+c_{2} \sinh \left\{\left(2 \alpha / 5 \beta a_{1}\right) \zeta\right\}}\right),
$$$$
\zeta= \pm \sqrt{\frac{\beta}{-12 \delta}} a_{1} x+\frac{16 \alpha^{2}}{75 \beta} t
$$ 
If we set $c_{2}=0$ and write $u(x, t)=v^{2}(x, t)$, then the above solutions can be written as

$$
\begin{aligned}
& u_{1}(x, t)=\frac{4 \alpha^{2}}{25 \beta^{2}}\left[-1 \pm \tanh \left(\frac{\alpha}{5 \sqrt{-3 \beta \delta}}\left[x+\frac{16 \alpha^{2}}{75 \beta} t\right]\right)\right]^{2}, \\
& u_{2}(x, t)=\frac{4 \alpha^{2}}{25 \beta^{2}}\left[1 \pm \tanh \left(\frac{\alpha}{5 \sqrt{-3 \beta \delta}}\left[x+\frac{16 \alpha^{2}}{75 \beta} t\right]\right)\right]^{2}, \\
& u_{3}(x, t)=\frac{4 \alpha^{2}}{25 \beta^{2}}\left[-1 \pm 2 \tanh \left(\frac{\alpha}{5 \sqrt{-3 \beta \delta}}\left[x+\frac{16 \alpha^{2}}{75 \beta} t\right]\right)\right]^{2} .
\end{aligned}
$$

Note that (35) is exactly the same solution of Khater and Hassan [35] as given in their first equation of (3.9) with $\zeta_{0}=0$. Similarly we can obtain the second solution of (36) in Hassan [5] if we set $c_{1}=0$ in our solution (35). The solution (35) represents kink shaped solitary and antikink shaped solitary solutions (depending upon the choice of sign) which are shown graphically in Figure 1 for the case $c_{1}=1$.

Case $A<0$ : (trigonometric type)

$$
\begin{gathered}
V_{1}(x, t)=-\frac{2 \alpha}{5 \beta}+\frac{2 \alpha i}{5 \beta}\left(\frac{-c_{1} \sin \{(i \lambda / 2) \zeta\}+c_{2} \cos \{(i \lambda / 2) \zeta\}}{c_{1} \cos \{(i \lambda / 2) \zeta\}+c_{2} \sin \{(i \lambda / 2) \zeta\}}\right), \\
\zeta= \pm \frac{2 \sqrt{1 /-75 \delta \beta} \alpha}{\lambda} x+\frac{16 \alpha^{2}}{75 \beta} t \\
V_{2}(x, t)=-\frac{2 \alpha}{5 \beta}-\frac{2 i \alpha}{5 \beta} \\
\times\left(\frac{-c_{1} \sin \{(i \lambda / 2) \zeta\}+c_{2} \cos \{(i \lambda / 2) \zeta\}}{c_{1} \cos \{(i \lambda / 2) \zeta\}+c_{2} \sin \{(i \lambda / 2) \zeta\}}\right) \\
\zeta= \pm \frac{2 \sqrt{1 /-75 \delta \beta} \alpha}{\lambda} x+\frac{16 \alpha^{2}}{75 \beta} t
\end{gathered}
$$

$V_{3}(x, t)$$$
=-\frac{2 \alpha}{5 \beta}+\frac{2 i \alpha}{5 \beta}
$$$$
\times\left(\frac{-c_{1} \sin \left\{\left(2 i \alpha / 5 \beta a_{1}\right) \zeta\right\}+c_{2} \cos \left\{\left(2 i \alpha / 5 \beta a_{1}\right) \zeta\right\}}{c_{1} \cos \left\{\left(2 i \alpha / 5 \beta a_{1}\right) \zeta\right\}+c_{2} \sin \left\{\left(2 i \alpha / 5 \beta a_{1}\right) \zeta\right\}}\right),
$$$$
\zeta= \pm \sqrt{\frac{\beta}{-12 \delta}} a_{1} x+\frac{16 \alpha^{2}}{75 \beta} t .
$$

But if $c_{2}=0$ and $u(x, t)=v^{2}(x, t)$, then trigonometric type solution becomes

$$
u_{1}(x, t)=\frac{4 \alpha^{2}}{25 \beta^{2}}\left[-1 \pm i \tan \left(\frac{\alpha}{5 \sqrt{3 \beta \delta}}\left[x+\frac{16 \alpha^{2}}{75 \beta} t\right]\right)\right]^{2},
$$

$$
\begin{aligned}
& u_{2}(x, t)=\frac{4 \alpha^{2}}{25 \beta^{2}}\left[1 \pm i \tan \left(\frac{\alpha}{5 \sqrt{3 \beta \delta}}\left[x+\frac{16 \alpha^{2}}{75 \beta} t\right]\right)\right]^{2}, \\
& u_{3}(x, t)=\frac{4 \alpha^{2}}{25 \beta^{2}}\left[-1 \pm 2 i \tan \left(\frac{\alpha}{5 \sqrt{3 \beta \delta}}\left[x+\frac{16 \alpha^{2}}{75 \beta} t\right]\right)\right]^{2}
\end{aligned}
$$

Case $A=0$ : (rational type)

$$
\begin{gathered}
u_{1}(x, t)=\frac{4 \alpha^{2}}{25 \beta^{2}}\left[-1+2\left(\frac{c_{2}}{c_{1}+c_{2} \zeta}\right)\right]^{2}, \\
u_{2}(x, t)=\frac{4 \alpha^{2}}{25 \beta^{2}}\left[1+2\left(\frac{c_{2}}{c_{1}+c_{2} \zeta}\right)\right]^{2}, \\
u_{3}(x, t)=\left[\frac{2 \alpha}{5 \beta}+a_{1}\left(\frac{c_{2}}{c_{1}+c_{2} \zeta}\right)\right]^{2} .
\end{gathered}
$$

As mentioned before, the $\left(G^{\prime} / G\right)$-expansion method gives more general types of solutions than that found by Khater and Hassan [35] and Hassan [5].

3.3. The Modified Two-Dimensional KP (Kadomtsev-Petviashvili) Equation. The modified KP equation (3) containing a square root nonlinearity is a very attractive model for the study of ion-acoustic waves in plasma physic [8]. We will obtain more general exact solutions of the modified KP equation. In order to find the traveling wave solution of (3), we let

$$
v(x, y, t)=v(\zeta), \quad \zeta=(x+k y-c t)
$$

Now, taking $u(x, y, t)=v^{2}(x, y, t)$, (3) becomes

$$
\begin{gathered}
\left(-c+\delta k^{2}\right) v v^{\prime \prime}+\left(-c+\delta k^{2}\right) v^{\prime 2}+\alpha v^{2} v^{\prime \prime}+2 \alpha v v^{\prime 2} \\
+\beta v v^{\prime \prime \prime \prime}+4 \beta v^{\prime} v^{\prime \prime \prime}+3 \beta\left(v^{\prime \prime}\right)^{2}=0,
\end{gathered}
$$

where $k, c, \beta, \delta$, and $\alpha$ are constants and the prime denotes differentiation with respect to $\zeta$. Integrating (41) with respect to $\zeta$ and setting the integration constant equal to zero, we obtain

$$
\left(-c+\delta k^{2}\right) v v^{\prime}+\alpha v^{2} v^{\prime}+3 \beta v^{\prime} v^{\prime \prime}+\beta v v^{\prime \prime \prime}=0 .
$$

Balancing $v^{2} v^{\prime}$ with $v v^{\prime \prime \prime}$ gives $m=2$. Therefore, we can write the solution of (42) in the form

$$
v(\zeta)=a_{0}+a_{1}\left(\frac{G^{\prime}}{G}\right)+a_{2}\left(\frac{G^{\prime}}{G}\right)^{2}
$$

where $a_{0}, a_{1}$, and $a_{2}$ are constants to be determined later. Substituting (43) along with (8) into (42) and collecting all terms with the same order of $\left(G^{\prime} / G\right)$, the left hand sides of (42) are converted into a polynomial in $\left(G^{\prime} / G\right)$. Setting each coefficient of each polynomial to zero, we derive a set of 


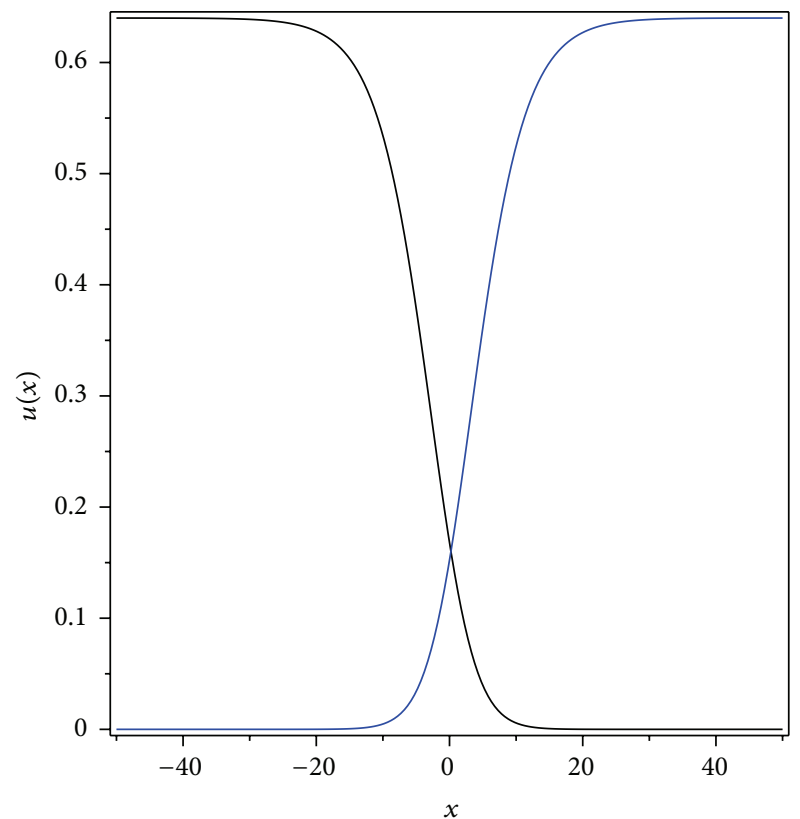

(a)

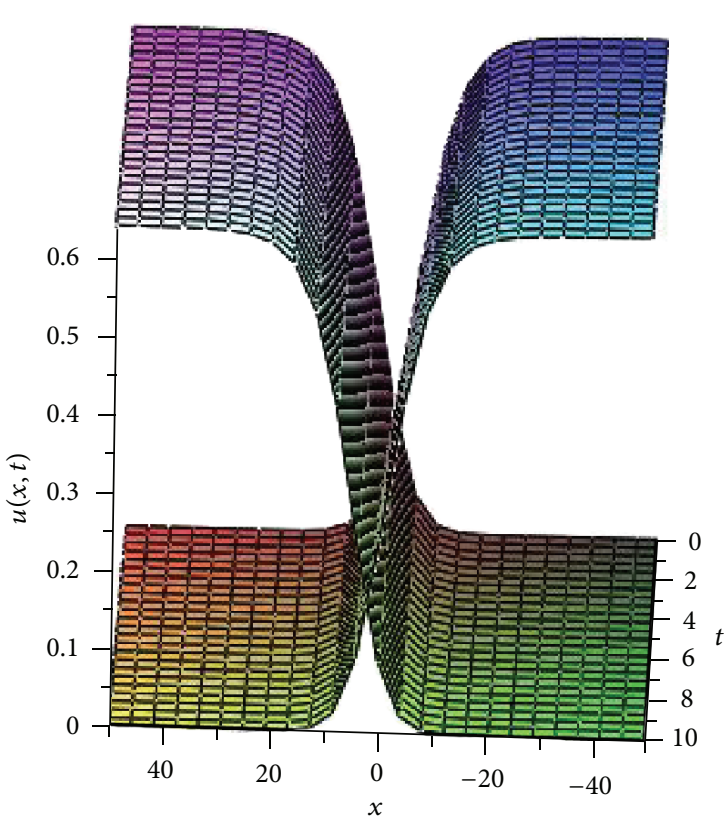

(b)

FIGURE 1: (a) 2D profile of (35): kink shaped solitary ( $u+$, blue line), anti-kink shaped solitary ( $u-$, black line). (b) Corresponding 3D plots when + sign is taken and when - ve sign is taken, with $\alpha=1, \beta=-1$, and $\delta=1$.

algebraic equations for $a_{0}, a_{1}, a_{2}, \delta, \lambda, \alpha, \beta, c, k$, and $\mu$ as follows:

$$
\begin{aligned}
& \left(\frac{G^{\prime}}{G}\right)^{7}:-60 \beta a_{2}^{2}-2 \alpha a_{2}^{3}=0, \\
& \left(\frac{G^{\prime}}{G}\right)^{6}:-2 \alpha a_{2}^{3} \lambda-5 \alpha a_{1} a_{2}^{2}-60 \beta a_{1} a_{2}-150 a_{2}^{2} \lambda=0 \text {, } \\
& \left(\frac{G^{\prime}}{G}\right)^{5}:-12 \beta a_{1}^{2}-5 \alpha a_{1} a_{2}^{2} \lambda-124 \beta a_{2}^{2} \lambda^{2}-2 k^{2} \delta a_{2}^{2} \\
& -24 \beta a_{0} a_{2}-4 \alpha a_{0} a_{2}-144 \beta a_{1} a_{2} \lambda+2 c a_{2}^{2} \\
& -4 \alpha a_{1}^{2} a_{2}-2 \alpha a_{2}^{3} \mu=0, \\
& \left(\frac{G^{\prime}}{G}\right)^{4}:-196 \beta a_{2}^{2} \lambda \mu-6 \alpha a_{0} a_{1} a_{2}-111 \beta a_{1} a_{2} \lambda^{2} \\
& -4 \alpha a_{0} a_{2}^{2} \lambda-32 \beta a_{2}^{2} \lambda^{3}-\alpha a_{1}^{3}-2 k^{2} \delta a_{2}^{2} \lambda+2 c a_{2}^{2} \lambda \\
& -54 \beta a_{0} a_{2} \lambda-5 \alpha a_{1} a_{2}^{2} \mu-4 \alpha a_{1}^{2} a_{2} \lambda+3 c a_{1} a_{2} \\
& -6 \beta a_{0} a_{1}-3 k^{2} \delta a_{1} a_{2}=0, \\
& \left(\frac{G^{\prime}}{G}\right)^{3}: 2 c a_{0} a_{2}-76 \beta a_{2}^{2} \mu^{2}-4 \alpha a_{0} a_{2}^{2} \mu-k^{2} \delta a_{1}^{2} \\
& -27 \beta a_{1} \lambda^{3} a_{2}-74 \beta a_{2}^{2} \lambda^{2} \mu+c a_{1}^{2}-6 \alpha a_{0} a_{1} a_{2} \lambda \\
& +3 c a_{1} a_{2} \lambda-40 \beta a_{0} a_{2} \mu-38 \beta a_{0} a_{2} \lambda^{2} \\
& -19 \beta a_{1}^{2} \lambda^{2}-2 \alpha a_{0} a_{1}^{2}-4 \alpha a_{1}^{2} a_{2} \mu
\end{aligned}
$$

$$
\begin{aligned}
& -168 \beta a_{1} a_{2} \lambda \mu-3 k^{2} \delta a_{1} a_{2} \lambda \\
& +2 c a_{2}^{2} \mu-2 \alpha a_{0}^{2} a_{2}-20 \beta a_{1}^{2} \mu-\alpha a_{1}^{3} \lambda \\
& -12 \beta a_{0} a_{1} \lambda-2 k^{2} \delta a_{0} a_{2}=0, \\
& \left(\frac{G^{\prime}}{G}\right)^{2}: 2 c a_{0} a_{2} \lambda-4 \beta a_{1}^{2} \lambda^{3}-8 \beta a_{0} a_{1} \mu \\
& -3 k^{2} \delta a_{1} a_{2} \mu-\alpha a_{1}^{3} \mu-52 \beta a_{0} a_{2} \lambda \mu+c a_{0} a_{1} \\
& +3 c a_{1} a_{2} \mu-6 \alpha a_{0} a_{1} a_{2} \mu-k^{2} \delta a_{1}^{2} \lambda \\
& -60 \beta a_{1} a_{2} \mu^{2}-\alpha a_{0}^{2} a_{1}-2 \alpha a_{0} a_{1}^{2} \lambda \\
& -2 k^{3} \delta a_{0} a_{2} \lambda-2 \alpha a_{0}^{2} a_{2} \lambda-7 \beta a_{0} a_{1} \lambda^{2} \\
& -26 \beta a_{1}^{2} \lambda \mu+c a_{1}^{2} \lambda-57 \beta a_{1} \lambda^{2} a_{2} \mu \\
& -8 \beta a_{0} a_{2} \lambda^{3}-k^{2} \delta a_{0} a_{1}-54 \beta a_{2}^{2} \lambda \mu^{2}=0, \\
& \left(\frac{G^{\prime}}{G}\right)^{1}:-2 \alpha a_{0}^{2} \mu+2 c a_{0} a_{2} \mu-\alpha a_{0}^{2} a_{1} \lambda-2 k^{2} \delta a_{0} a_{2} \mu \\
& -8 \beta a_{1}^{2} \mu^{2}+c a_{0} a_{1} \lambda-16 \beta a_{0} a_{2} \mu^{2} \\
& -8 \beta a_{0} a_{1} \lambda \mu-\beta a_{0} a_{1} \lambda^{3}-14 \beta a_{0} a_{2} \lambda^{2} \mu \\
& -36 \beta \lambda \mu^{2} a_{1} a_{2}-12 \beta a_{2}^{2} \mu^{3}-k^{2} \delta a_{0} a_{1} \lambda \\
& -k^{2} \delta a_{1}^{2} \mu+c a_{1}^{2} \mu-2 \alpha a_{0} a_{1}^{2} \mu-7 \beta \mu \lambda^{2} a_{1}^{2}=0,
\end{aligned}
$$




$$
\begin{aligned}
\left(\frac{G^{\prime}}{G}\right)^{0}: & -6 \beta \mu^{3} a_{1} a_{2}+c a_{0} a_{1} \mu-\alpha \mu a_{0}^{2} a_{1}-3 \beta \lambda \mu^{2} a_{1}^{2} \\
& -\beta \mu \lambda^{2} a_{0} a_{1}-6 \beta \lambda \mu^{2} a_{0} a_{2}-2 \beta a_{0} a_{1} \mu^{2} \\
& -k^{2} \delta \mu a_{0} a_{1}=0 .
\end{aligned}
$$

Solving this system by Maple gives two sets of solutions.

Case 1. We have

$$
\begin{gathered}
a_{0}=\frac{-30 \beta \mu}{\alpha}, \quad a_{1}=\frac{-30 \beta \lambda}{\alpha}, \quad a_{2}=\frac{-30 \beta}{\alpha}, \\
c=-16 \beta \mu+4 \beta \lambda^{2}+k^{2} \delta .
\end{gathered}
$$

Substituting the above case and the general solution (8) into (43) and according to (42), we obtain three types of traveling wave solutions of (3) as follows.

If $A>0$, we have the hyperbolic type

$v(x, y, t)$

$$
\begin{aligned}
& =\frac{-30 \beta \mu}{\alpha}-\frac{30 \beta \lambda}{\alpha} \\
& \times\left[\frac{-\lambda}{2}+\frac{\sqrt{A}}{2}\right. \\
& \left.\quad \times\left(\frac{c_{1} \sinh \{(\sqrt{A} / 2) \zeta\}+c_{2} \cosh \{(\sqrt{A} / 2) \zeta\}}{c_{1} \cosh \{(\sqrt{A} / 2) \zeta\}+c_{2} \sinh \{(\sqrt{A} / 2) \zeta\}}\right)\right] \\
& -\frac{30 \beta}{\alpha}\left[\frac{-\lambda}{2}+\frac{\sqrt{A}}{2}\right. \\
& \left.\quad \times\left(\frac{c_{1} \sinh \{(\sqrt{A} / 2) \zeta\}+c_{2} \cosh \{(\sqrt{A} / 2) \zeta\}}{c_{1} \cosh \{(\sqrt{A} / 2) \zeta\}+c_{2} \sinh \{(\sqrt{A} / 2) \zeta\}}\right)\right]^{2} .
\end{aligned}
$$

In particular, if $c_{1} \neq 0, c_{2}=0, \lambda>0$, and $\mu=0$, then $u(x, y, t)$ becomes

$$
\begin{array}{r}
u(x, y, t)=\frac{225 \beta^{2} \lambda^{4}}{4 \alpha^{2}} \operatorname{sech}^{4}\left\{\frac{\lambda}{2} \zeta\right\}, \\
\zeta=x+k y-\left(4 \beta \lambda^{2}+k^{2} \delta\right) t .
\end{array}
$$

If $A<0$, we have the trigonometric type

$$
\begin{aligned}
& v(x, y, t) \\
& =\frac{-30 \beta \mu}{\alpha}-\frac{30 \beta \lambda}{\alpha} \\
& \quad \times\left[\frac{-\lambda}{2}+\frac{\sqrt{A}}{2}\right. \\
& \left.\quad \times\left(\frac{-c_{1} \sin \{(\sqrt{A} / 2) \zeta\}+c_{2} \cos \{(\sqrt{A} / 2) \zeta\}}{c_{1} \cos \{(\sqrt{A} / 2) \zeta\}+c_{2} \sin \{(\sqrt{A} / 2) \zeta\}}\right)\right]
\end{aligned}
$$

$$
\begin{aligned}
-\frac{30 \beta}{\alpha}[ & \frac{-\lambda}{2}+\frac{\sqrt{A}}{2} \\
& \left.\times\left(\frac{-c_{1} \sin \{(\sqrt{A} / 2) \zeta\}+c_{2} \cos \{(\sqrt{A} / 2) \zeta\}}{c_{1} \cos \{(\sqrt{A} / 2) \zeta\}+c_{2} \sin \{(\sqrt{A} / 2) \zeta\}}\right)\right]^{2} .
\end{aligned}
$$

So, the traveling wave solutions of (3) in this case are

$$
\begin{gathered}
u(x, y, t)=\frac{225 \beta^{2} \lambda^{4}}{4 \alpha^{2}} \sec ^{4}\left\{\frac{\sqrt{-\lambda^{2}}}{2} \zeta\right\}, \\
\zeta=x+k y-\left(4 \beta \lambda^{2}+k^{2} \delta\right) t
\end{gathered}
$$

Case 2. We have

$$
\begin{gathered}
a_{0}=\frac{-5 \beta\left(\lambda^{2}+2 \mu\right)}{\alpha}, \quad a_{1}=\frac{-30 \beta \lambda}{\alpha}, \quad a_{2}=\frac{-30 \beta}{\alpha}, \\
c=16 \beta \mu-4 \beta \lambda^{2}+k^{2} \delta .
\end{gathered}
$$

If $A>0$, we have the hyperbolic type

$$
\begin{aligned}
& v(x, y, t) \\
& =-\frac{5 \beta\left(\lambda^{2}+2 \mu\right)}{\alpha}-\frac{30 \beta \lambda}{\alpha} \\
& \times\left[\frac{-\lambda}{2}+\frac{\sqrt{A}}{2}\right. \\
& \left.\quad \times\left(\frac{c_{1} \sinh \{(\sqrt{A} / 2) \zeta\}+c_{2} \cosh \{(\sqrt{A} / 2) \zeta\}}{c_{1} \cosh \{(\sqrt{A} / 2) \zeta\}+c_{2} \sinh \{(\sqrt{A} / 2) \zeta\}}\right)\right] \\
& -\frac{30 \beta}{\alpha}\left[\frac{-\lambda}{2}+\frac{\sqrt{A}}{2}\right. \\
& \left.\quad \times\left(\frac{c_{1} \sinh \{(\sqrt{A} / 2) \zeta\}+c_{2} \cosh \{(\sqrt{A} / 2) \zeta\}}{c_{1} \cosh \{(\sqrt{A} / 2) \zeta\}+c_{2} \sinh \{(\sqrt{A} / 2) \zeta\}}\right)\right]^{2} .
\end{aligned}
$$

However, if $c_{1} \neq 0, c_{2}=0, \lambda>0$, and $\mu=0$, then $u(x, y, t)$ becomes

$$
\begin{gathered}
u(x, y, t)=\frac{25 \beta^{2} \lambda^{4}}{4 \alpha^{2}}\left[2-3 \operatorname{sech}^{2}\left\{\frac{\lambda}{2} \zeta\right\}\right]^{2} \\
\zeta=x+k y-\left(-4 \beta \lambda^{2}+k^{2} \delta\right) t
\end{gathered}
$$

If $A<0$, we have the trigonometric type

$v(x, y, t)$

$$
=-\frac{5 \beta\left(\lambda^{2}+2 \mu\right)}{\alpha}-\frac{30 \beta \lambda}{\alpha}
$$




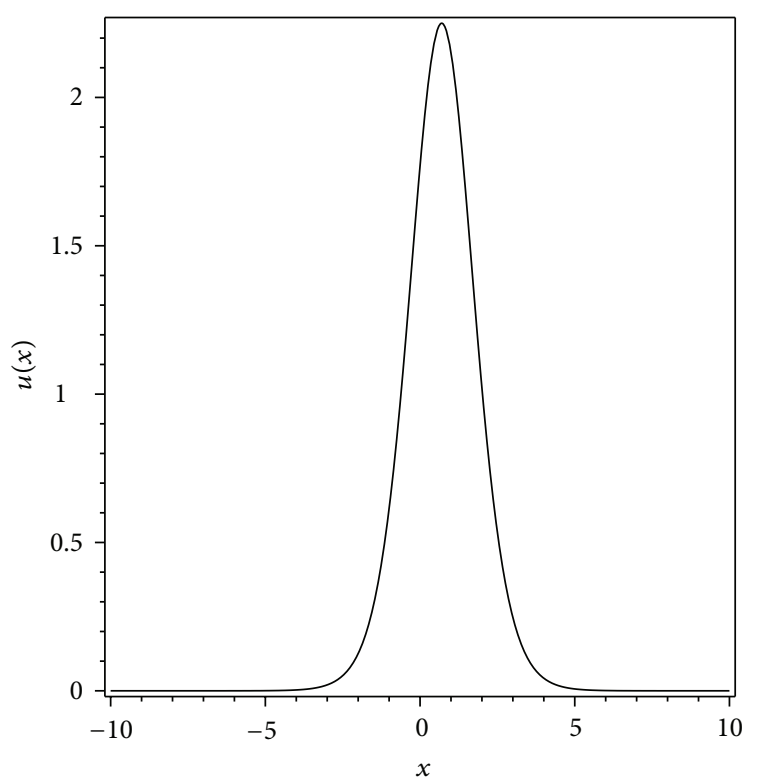

(a)

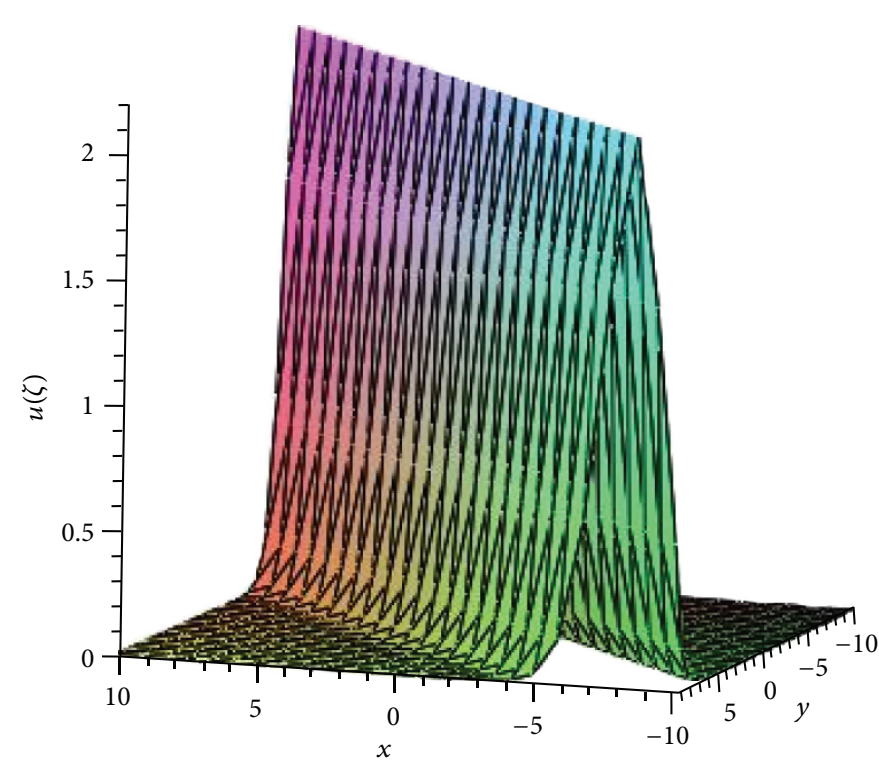

(b)

FIGURE 2: Bell type solitary (a) 2D profile and (b) corresponding 3D plot of (47) for parameters $\alpha=2, \beta=0.4$ and $\delta=0.1, \lambda=1, k=1$, and $t=0.5$.

$$
\begin{aligned}
& \times\left[\frac{-\lambda}{2}+\frac{\sqrt{A}}{2}\right. \\
& \left.\quad \times\left(\frac{-c_{1} \sin \{(\sqrt{A} / 2) \zeta\}+c_{2} \cos \{(\sqrt{A} / 2) \zeta\}}{c_{1} \cos \{(\sqrt{A} / 2) \zeta\}+c_{2} \sin \{(\sqrt{A} / 2) \zeta\}}\right)\right] \\
& -\frac{30 \beta}{\alpha}\left[\frac{-\lambda}{2}+\frac{\sqrt{A}}{2}\right. \\
& \left.\quad \times\left(\frac{-c_{1} \sin \{(\sqrt{A} / 2) \zeta\}+c_{2} \cos \{(\sqrt{A} / 2) \zeta\}}{c_{1} \cos \{(\sqrt{A} / 2) \zeta\}+c_{2} \sin \{(\sqrt{A} / 2) \zeta\}}\right)\right]^{2} .
\end{aligned}
$$

In the particular case when $c_{1} \neq 0, c_{2}=0, \lambda>0$, and $\mu=0$, $u(x, y, t)$ becomes

$$
\begin{gathered}
u(x, y, t)=\frac{25 \beta^{2} \lambda^{4}}{4 \alpha^{2}}\left[2-3 \sec ^{2}\left\{\frac{\sqrt{-\lambda^{2}}}{2} \zeta\right\}\right]^{2}, \\
\zeta=x+k y-\left(-4 \beta \lambda^{2}+k^{2} \delta\right) t .
\end{gathered}
$$

If $A=0$, we have the rational type

$$
\begin{aligned}
v(x, y, t)= & -\frac{5 \beta\left(\lambda^{2}+2 \mu\right)}{\alpha}-30 k^{2} \delta \lambda\left[-\frac{\lambda}{2}+\left(\frac{c_{2}}{c_{1}+c_{2} \zeta}\right)\right] \\
& -30 k^{2} \delta\left[-\frac{\lambda}{2}+\left(\frac{c_{2}}{c_{1}+c_{2} \zeta}\right)\right]^{2},
\end{aligned}
$$

where $\zeta=x+k y-\left(16 \beta \mu-4 \beta \lambda^{2}+k^{2} \delta\right) t$.
We remark that our results in (47) and (52), when $c_{1} \neq 0$, $c_{2}=0, \lambda>0$, and $\mu=0$, match those of Khater et al. [6] (2.19) when $a=1$. In Figure 2, we plot the bell type solitary for 2D profile and the corresponding 3D plot of (47) for parameters $\alpha=2, \beta=0.4$ and $\delta=0.1, \lambda=1, k=1$, and $t=0.5$.

\section{Conclusion}

The $\left(G^{\prime} / G\right)$-expansion was applied to solve the model of ionacoustic waves in plasma physics where these equations each contain a square root nonlinearity. The $\left(G^{\prime} / G\right)$-expansion has been successfully used to obtain some exact traveling wave solutions of the Schamel equation, Schamel-KdV (S$\mathrm{KdV}$ ) equation, and modified KP (Kadomtsev-Petviashvili) equation. Moreover, the reliability of the method and the reduction in the size of computational domain give this method a wider applicability. This fact shows that our algorithm is effective and more powerful for NLPDE. In all the general solutions (22), (35), (47), and (52), we have the additional arbitrary constants $c_{1}, c_{2}, \lambda$, and $\mu$. We note that the special case $c_{1} \neq 0, c_{2}=0, \lambda>0$, and $\mu=0$ reproduced the results of Khater and Hassan [35], Hassan [5] and Khater et al. [6]. Many different new forms of traveling wave solutions such as the kink shaped, antikink shaped, and bell type solitary solutions were obtained. Finally, numerical simulations are given to complete the study.

Moreover, all the methods have some limitations in their applications. In fact, there is no unified method that can be used to handle all types of nonlinear partial differential equations (NLPDE). Certainly, each investigator in the field of differential equations has his own experience to choose the method depending on form of the nonlinear differential equation and the pole of its solution. So, the limitations of 
the $\left(G^{\prime} / G\right)$-expansion method used a rise only when the equation has the traveling wave and becomes powerful in finding traveling wave solutions of NLPDE only.

In our future works, we can extend our method by introducing a more generalized ansätz $G^{\prime 2}=d_{2} G^{2}+d_{3} G^{3}+$ $d_{4} G^{4}$, where $G=G(\zeta)$, to solve Schamel equation, Schamel$\mathrm{KdV}(\mathrm{S}-\mathrm{KdV}$ ) equation, and modified Kadomtsev-Petviashvili (KP) equation.

\section{Acknowledgment}

This work is financially supported by Universiti Kebangsaan Malaysia Grant: UKM-DIP-2012-31.

\section{References}

[1] G. B. Whitham, Linear and Nonlinear Waves, Pure and Applied Mathematics, John Wiley \& Sons, New York, NY, USA, 1974.

[2] R. C. Davidson, Methods in Nonlinear Plasma Theory, Academic Press, New York, NY, USA, 1972.

[3] H. Schamel, "A modified Korteweg de Vries equation for ion acoustic waves due to resonant electrons," Journal of Plasma Physics, vol. 9, pp. 377-387, 1973.

[4] J. Lee and R. Sakthivel, "Exact travelling wave solutions of the Schamel-Korteweg-de Vries equation," Reports on Mathematical Physics, vol. 68, no. 2, pp. 153-161, 2011.

[5] M. M. Hassan, "New exact solutions of two nonlinear physical models," Communications in Theoretical Physics, vol. 53, no. 4, pp. 596-604, 2010.

[6] A. H. Khater, M. M. Hassan, and D. K. Callebaut, “Travelling wave solutions to some important equations of mathematical physics," Reports on Mathematical Physics, vol. 66, no. 1, pp. 119,2010

[7] D. Chakraborty and K. P. Das, "Stability of ion-acoustic solitons in a multispecies plasma consisting of non-isothermal electrons," Journal of Plasma Physics, vol. 60, no. 1, pp. 151-158, 1998.

[8] B. B. Kadomtsev and V. I. Petviashvili, "On the stability of solitary in weakly dispersive media," Soviet Physics Doklady, vol. 15, pp. 539-541, 1970.

[9] M. Krisnangkura, S. Chinviriyasit, and W. Chinviriyasit, "Analytic study of the generalized Burger's-Huxley equation by hyperbolic tangent method," Applied Mathematics and Computation, vol. 218, no. 22, pp. 10843-10847, 2012.

[10] A.-M. Wazwaz, "The tanh method for traveling wave solutions of nonlinear equations," Applied Mathematics and Computation, vol. 154, no. 3, pp. 713-723, 2004.

[11] Q. Shi, Q. Xiao, and X. Liu, "Extended wave solutions for a nonlinear Klein-Gordon-Zakharov system," Applied Mathematics and Computation, vol. 218, no. 19, pp. 9922-9929, 2012.

[12] A.-M. Wazwaz, "The tanh and the sine-cosine methods for a reliable treatment of the modified equal width equation and its variants," Communications in Nonlinear Science and Numerical Simulation, vol. 11, no. 2, pp. 148-160, 2006.

[13] A. Jabbari and H. Kheiri, "New exact traveling wave solutions for the Kawahara and modified Kawahara equations by using modified tanh-coth method," Acta Universitatis Apulensis, no. 23, pp. 21-38, 2010.

[14] K. Parand and J. A. Rad, "Exp-function method for some nonlinear PDE's and a nonlinear ODE's," Journal of King Saud University-Science, vol. 24, no. 1, pp. 1-10, 2012.
[15] M. K. Elboree, "New soliton solutions for a KadomtsevPetviashvili (KP) like equation coupled to a Schrödinger equation," Applied Mathematics and Computation, vol. 218, no. 10, pp. 5966-5973, 2012.

[16] A. S. Abdel Rady, E. S. Osman, and M. Khalfallah, "On soliton solutions of the $(2+1)$ dimensional Boussinesq equation," Applied Mathematics and Computation, vol. 219, no. 8, pp. 34143419, 2012.

[17] B. Hong and D. Lu, "New Jacobi elliptic function-like solutions for the general KdV equation with variable coefficients," Mathematical and Computer Modelling, vol. 55, no. 3-4, pp. 1594-1600, 2012.

[18] J. Lee, R. Sakthivel, and L. Wazzan, "Exact traveling wave solutions of a higher-dimensional nonlinear evolution equation," Modern Physics Letters B, vol. 24, no. 10, pp. 1011-1021, 2010.

[19] S. Abbasbandy and A. Shirzadi, "The first integral method for modified Benjamin-Bona-Mahony equation," Communications in Nonlinear Science and Numerical Simulation, vol. 15, no. 7, pp. 1759-1764, 2010.

[20] N. Taghizadeh, M. Mirzazadeh, and F. Tascan, "The firstintegral method applied to the Eckhaus equation," Applied Mathematics Letters, vol. 25, no. 5, pp. 798-802, 2012.

[21] W. Malfliet, "Solitary wave solutions of nonlinear wave equations," American Journal of Physics, vol. 60, no. 7, pp. 650-654, 1992.

[22] M. Wang, X. Li, and J. Zhang, "The $\left(G^{\prime} / G\right)$-expansion method and travelling wave solutions of nonlinear evolution equations in mathematical physics," Physics Letters A, vol. 372, no. 4, pp. 417-423, 2008.

[23] W. M. Taha and M. S. M. Noorani, "Exact solutions of equation generated by the Jaulent-Miodek hierarchy by $\left(G^{\prime} / G\right)$ expansion method," Mathematical Problems in Engineering, vol. 2013, Article ID 392830, 7 pages, 2013.

[24] W. M. Taha, M. S. M. Noorani, and I. Hashim, "New application of the $\left(G^{\prime} / G\right)$-expansion method for thin film equations," Abstract and Applied Analysis, vol. 2013, Article ID 535138, 6 pages, 2013.

[25] B. Ayhan and A. Bekir, "The $\left(G^{\prime} / G\right)$-expansion method for the nonlinear lattice equations," Communications in Nonlinear Science and Numerical Simulation, vol. 17, no. 9, pp. 3490-3498, 2012.

[26] J. Feng, W. Li, and Q. Wan, "Using $\left(G^{\prime} / G\right)$-expansion method to seek the traveling wave solution of Kolmogorov-PetrovskiiPiskunov equation," Applied Mathematics and Computation, vol. 217, no. 12, pp. 5860-5865, 2011.

[27] M. M. Kabir, A. Borhanifar, and R. Abazari, "Application of $\left(G^{\prime} / G\right)$-expansion method to regularized long wave (RLW) equation," Computers \& Mathematics with Applications, vol. 61, no. 8, pp. 2044-2047, 2011.

[28] A. Malik, F. Chand, H. Kumar, and S. C. Mishra, "Exact solutions of the Bogoyavlenskii equation using the multiple $\left(G^{\prime} / G\right)$-expansion method," Computers \& Mathematics with Applications, vol. 64, no. 9, pp. 2850-2859, 2012.

[29] A. Malik, F. Chand, and S. C. Mishra, "Exact travelling wave solutions of some nonlinear equations by $\left(G^{\prime} / G\right)$-expansion method," Applied Mathematics and Computation, vol. 216, no. 9, pp. 2596-2612, 2010.

[30] A. Jabbari, H. Kheiri, and A. Bekir, "Exact solutions of the coupled Higgs equation and the Maccari system using He's semiinverse method and $\left(G^{\prime} / G\right)$-expansion method," Computers \& Mathematics with Applications, vol. 62, no. 5, pp. 2177-2186, 2011. 
[31] H. Naher and F. A. Abdullah, "Some new traveling wave solutions of the nonlinear reaction diffusion equation by using the improved $\left(G^{\prime} / G\right)$-expansion method," Mathematical Problems in Engineering, vol. 2012, Article ID 871724, 17 pages, 2012.

[32] E. M. E. Zayed and M. A. M. Abdelaziz, "The two-variable $\left(G^{\prime} / G, 1 / G\right)$-expansion method for solving the nonlinear KdV$\mathrm{mKdV}$ equation," Mathematical Problems in Engineering, vol. 2012, Article ID 725061, 14 pages, 2012.

[33] H. Naher and F. A. Abdullah, “The improved $\left(G^{\prime} / G\right)$-expansion method for the $(2+1)$-dimensional modified ZakharovKuznetsov equation," Journal of Applied Mathematics, vol. 2012, Article ID 438928, 20 pages, 2012.

[34] H. Naher and F. A. Abdullah, "New traveling wave solutions by the extended generalized Riccati equation mapping method of the $(2+1)$-dimensional evolution equation," Journal of Applied Mathematics, vol. 2012, Article ID 486458, 18 pages, 2012.

[35] A. H. Khater and M. M. Hassan, "Exact solutions expressible in hyperbolic and jacobi elliptic functions of some important equations of ion-acoustic waves," in Acoustic Waves-From Microdevices to Helioseismology, 2011. 


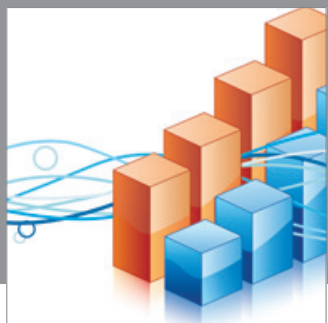

Advances in

Operations Research

mansans

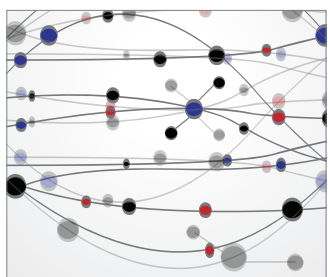

The Scientific World Journal
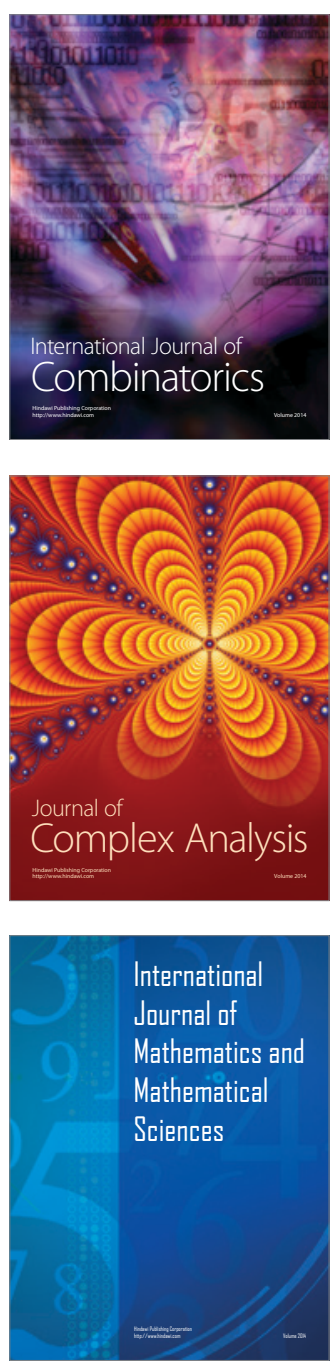
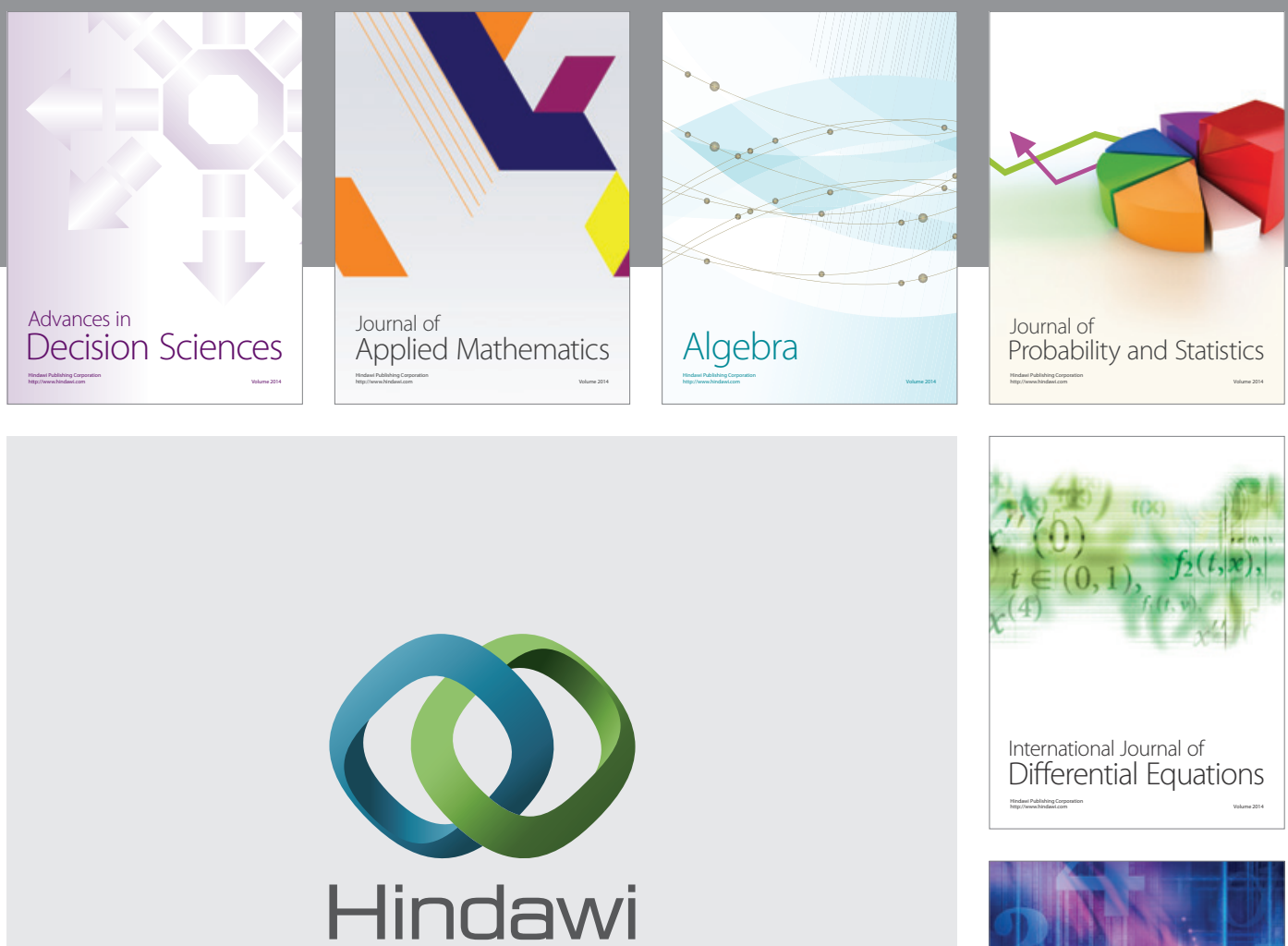

Submit your manuscripts at http://www.hindawi.com
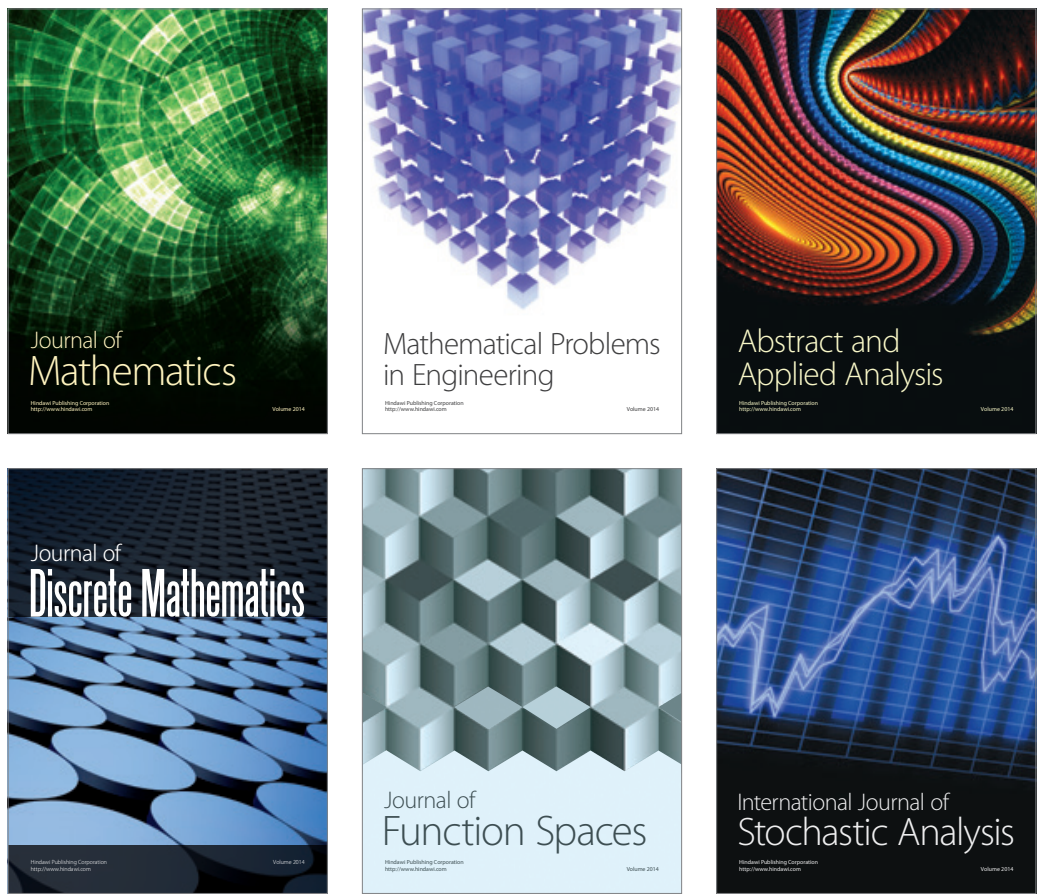

Journal of

Function Spaces

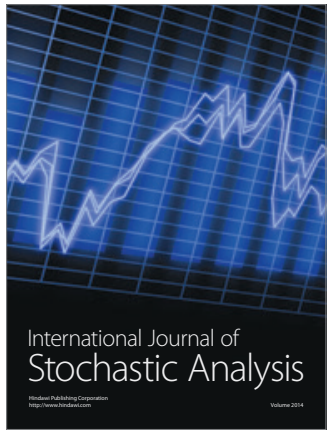

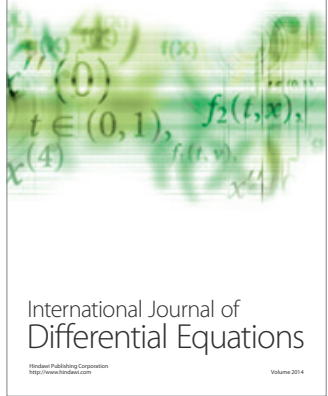
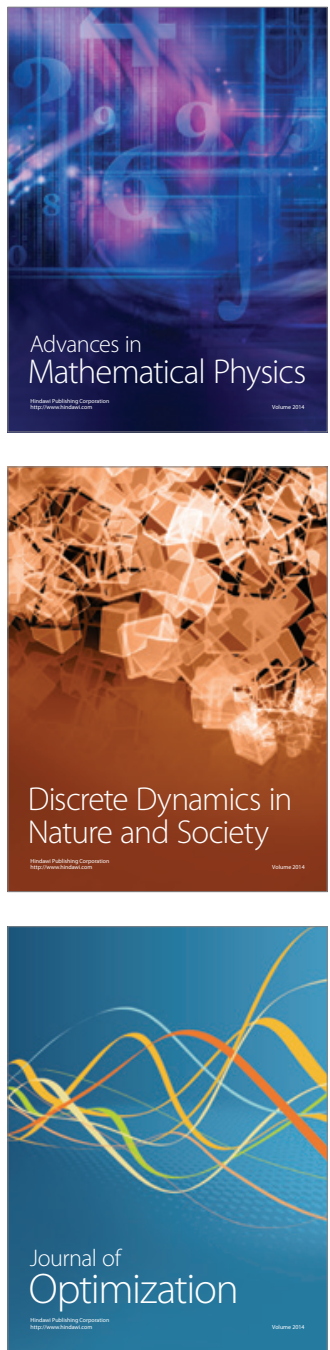